\title{
Long-Term Facilitation of Amphetamine-Induced Rotational Behavior and Striatal Dopamine Release Produced by a Single Exposure to Amphetamine: Sex Differences
}

\author{
TERRY E. ROBINSON, JILL B. BECKER and SHARON K. PRESTY \\ Psychology Department and Neuroscience Laboratory, University of Michigan, 1103 E. Huron St., Ann Arbor, \\ MI 48109 (U.S.A.)
}

(Accepted May 24th, 1982)

Key words: long-term facilitation — amphetamine — rotational behavior — striatal dopamine

\begin{abstract}
Amphetamine (AMPH)-induced rotational behavior in non-lesioned rats and AMPH-stimulated dopamine (DA) release from striatal tissue fragments in vitro were used to study the long-term effects of a single injection of AMPH on activity in the mesostriatal DA system. A single injection of a low dose of AMPH $(1.25 \mathrm{mg} / \mathrm{kg})$ greatly enhanced the rotational behavior produced by a second injection of AMPH given 3-4 weeks later in intact female, ovariectomized female and castrated male rats. The effect of AMPH pretreatment in intact males differed from that in the other groups. When only 7-8 days separated the two test sessions both intact male and female rats showed sensitization of rotational behavior, but the magnitude of the change was greater in females. In addition, a single injection of $1.25 \mathrm{mg} / \mathrm{kg}$ of AMPH in vivo produced a long-lasting (3-5 weeks) enhancement of AMPH-stimulated DA release from striatal tissue in vitro. It is suggested that: (1) repeated injections of AMPH are not necessary to produce a longlasting facilitation of behaviors mediated by the mesostriatal DA system; (2) gender and/or hormonal state influences the development of long-term changes in the mesostriatal DA system; and (3) changes in DA release from presynaptic terminals may contribute to the behavioral sensitization produced by stimulant drugs. The phenomena reported here may provide complementary in vitro and in vivo models for studying neuroplasticity in brain DA systems.
\end{abstract}

\section{INTRODUCTION}

It is well established that the repeated administration of amphetamine (AMPH) results in a progressively enhanced behavioral response to subsequent injections $11,22,26,39,41,44$. That is, the locomotion, stereotypy or rotational behavior produced by the initial injection of AMPH typically has a less rapid onset, is less intense, and/or is shorter-lasting than observed following subsequent injections. This phenomenon has been described in different species, with a variety of stimulant drugs, and has been variously termed sensitization, reverse tolerance, agonist hypersensitivity and behavioral facilitation. Although many different paradigms have been used to study sensitization the typical experiment involves a series of injections of a dopamine mimetic drug, usually given one or two times a day, and uses stereotypy as the behavioral index of mesostriatal DA activity.
However, it is not clear that dopamine-mimetic drugs must be administered repeatedly to produce long-term changes in behaviors mediated by the mesostriatal DA system. A single injection of AMPH or apomorphine may enhance the behavioral effects of a subsequent injection given weeks later $2,7,12,27$. In fact, it has been suggested that the intermittent exposure to stimulants may be more effective in producing sensitization than repeated or continuous exposure $1,12,31,33$. In the experiments reported here we examined the long-term effects of a single injection of AMPH on AMPH-induced rotational behavior. Since we had previously found that gonadal hormones modulate mesostriatal DA activity in a sexually dimorphic manner ${ }^{6,36}$, we also examined the influence of gonadectomy in both males and females.

Another question of interest concerns the neurological basis of sensitizaticn. A number of studies have examined the effects of repeated injections of 
AMPH on DA receptor binding, but with conflicting results $17,19,21,30,34$. Studies of DA levels, synthesis and metabolism have also failed to find evidence for long-lasting changes which could account for behavioral sensitization 24,40 . We approached this problem by examining the long-term effects of a single injection of AMPH on the AMPH-stimulated release of DA from striatal tissue in vitro ${ }^{4}$. We found that a single exposure to $\mathrm{AMPH}$ in vivo produced a long-term enhancement of AMPH-stimulated DA release in vitro. These changes in DA release parallel the sensitization of rotational behavior, and therefore may be at least partially responsible for the phenomenon of sensitization.

\section{MATERIALS AND METHODS}

\section{Subjects}

The experiments were conducted with $250-350 \mathrm{~g}$ adult Holtzman rats (Holtzman, Madison, WI), which were housed individually and had constant access to food and water. The animal colony was maintained on a reversed light/dark cycle $(14: 10 \mathrm{~h})$ with the lights going out at $06.30 \mathrm{~h}$. All testing was conducted during the 'night' portion of the rats' day/night cycle.

\section{Procedures for testing rotational behavior}

We used amphetamine (AMPH)-induced rotational behavior in non-lesioned rats as a behavioral index of mesostriatal dopamine (DA) activity ${ }^{14}$. Amphetamine-induced rotational behavior in rats with unilateral 6-hydroxydopamine lesions of the mesostriatal DA system is thought to be due to the asymmetrical release of DA in the striatum ${ }^{43}$. When AMPH is administered to non-lesioned rats they also turn in circles in one dominant direction ${ }^{14,20}$. This behavioral asymmetry observed in normal animals is thought to be due to an endogenous asymmetry in the mesostriatal DA system ${ }^{14}$.

Rotational behavior was measured by automated spherical rotometers similar to those described by Greenstein and Glick ${ }^{16}$. The rotometers were interfaced with a microprocessor which recorded every $1 / 4$ turn $\left(90^{\circ}\right)$ made to the right or left during each $5 \mathrm{~min}$ interval. From these data the following were calculated: (a) total $1 / 4$ turns - the number of $1 / 4$ turns made to the left plus those made to the right; (b) total rotations - a rotation consists of 4 consecutive $1 / 4$ turns in the same direction. Total rotations are rotations to the left plus those to the right; (c) net rotations - net rotations are the number of total rotations in the dominant direction minus those in the non-dominant direction; (d) percent dominance - the number of total rotations in the dominant direction divided by total rotations times 100 (i.e. the percent of total rotations made in the dominant direction); and (e) extra $1 / 4$ turns - the number of total $1 / 4$ turns minus total rotations times 4 (i.e. the number of $1 / 4$ turns which do not contribute to rotations).

In our initial experiment all animals were gently handled for a few days prior to testing. On the first day of testing they were placed in a rotometer for a 15-min habituation period. Immediately following this each animal was injected intraperitoneally (i.p.) with $1.25 \mathrm{mg} / \mathrm{kg}$ of $\mathrm{D}$ amphetamine sulfate (AMPH) dissolved in $0.9 \%$ saline $(1.25 \mathrm{mg} / \mathrm{ml})$. Following the injection rotational behavior was recorded for $60 \mathrm{~min}$, after which time the animal was removed from the rotometer and replaced in its home cage. The animals were then left undisturbed in their home cages for 3-4 weeks. After 3-4 weeks the above procedure was repeated.

The dose of $1.25 \mathrm{mg} / \mathrm{kg}$ of AMPH was selected on the basis of the extensive dose-response studies of Glick and his colleagues ${ }^{14}$. These researchers have examined the rotational behavior of non-lesioned rats given over 12 different doses of $\mathrm{AMPH}$, ranging from 0.625 to $25 \mathrm{mg} / \mathrm{kg}$. They found a biphasic dose-response with peak rotational behavior occurring at 1.25 and $20.0 \mathrm{mg} / \mathrm{kg}$. The $20 \mathrm{mg} / \mathrm{kg}$ dose produced more variable rotational behavior than $1.25 \mathrm{mg} / \mathrm{kg}$, and doses between 5 and $15 \mathrm{mg} / \mathrm{kg}$ resulted in relatively poor rotational behavior (see ref. 14 for a discussion of this issue). Therefore, to use rotational behavior in non-lesioned rats as a behavioral index of mesostriatal DA activity one is confined to a narrow effective dose range.

In previous studies we found sex differences in both AMPH-induced rotational behavior and the AMPH-stimulated release of DA from striatal tissue $^{5,6,35}$. Since gonadal hormones appear to modulate mesostriatal DA activity we tested 4 groups of rats in this experiment: (1) intact females; (2) intact 
males; (3) ovariectomized females (OVX); and (4) castrated males (CAST). Gonadectomies or shamoperations were performed under ether anaesthesia at least 2 weeks prior to the first test session. One additional group of intact female rats was tested in the same manner as described above; however, during the first test session saline was injected and during the second test session ( 3 weeks later) 1.25 $\mathrm{mg} / \mathrm{kg}$ of AMPH was administered.

The second behavioral experiment reported here was conducted exactly as described above, except: (1) only intact male and female rats were tested; (2) there were only 7-8 days between the first and second test sessions; and (3) the males were tested with $1.25 \mathrm{mg} / \mathrm{kg}$ and the females with $0.85 \mathrm{mg} / \mathrm{kg}$ of AMPH. We know from a previous study that these systemic doses of AMPH result in equivalent brain levels of AMPH in male and female rats ${ }^{5}$.

\section{AMPH-stimulated release of striatal $D A$ in vitro}

This experiment was conducted with $16 \mathrm{OVX}$ female rats. At least 2 weeks after OVX, half of the rats were given an i.p. injection of $1.25 \mathrm{mg} / \mathrm{kg}$ of AMPH dissolved in $0.9 \%$ saline and the other half an injection of an equal volume of saline. The animals were immediately replaced in their home cages and remained there undisturbed for 3-5 weeks. At the end of this time the animals were killed by decapitation and the left and right striatum quickly removed from the brain. The two striata from each rat were pooled and placed in an individual perifusion chamber. The perifusion chamber was positioned in a constant temperature $\left(37^{\circ} \mathrm{C}\right)$ water bath. See Becker and Ramirez $z^{4}$ and Gallardo and Ramirez ${ }^{13}$ for a detailed description of the perifusion apparatus and technique. Briefly, a medium consisting of a Krebs-phosphate buffer also containing $0.1 \%$ albumin and $18 \% \mathrm{D}$-glucose at $\mathrm{pH}$ 7.4 was infused through the chamber at a constant rate of $100 \mu \mathrm{l} / \mathrm{min}$, and a volume of $500 \mu \mathrm{l}$ maintained in the chamber. Following a $45-\mathrm{min}$ equilibration period effluent samples were collected on ice at 4 min intervals. As each sample was collected, 20 $\mu \mathrm{l}$ of a solution containing $8 \times 10^{-4} \mathrm{M} \mathrm{NaHSO}_{4}$ and $4 \mathrm{mg} / \mathrm{l}$ dihydroxybenzylamine in $0.05 \mathrm{~N} \mathrm{HClO}_{4}$ was added to each tube. Following the collection of the 5 baseline samples, a pulse of $D$-amphetamine (initial salt concentration in the chamber of $10^{-3} \mathrm{M}$ ) was delivered into the chamber and samples collected at $4 \mathrm{~min}$ intervals for an additional $20 \mathrm{~min}$. Samples were centrifuged at $3500 \times g$ for $45 \mathrm{~min}$ at $4{ }^{\circ} \mathrm{C}$ after collection. These effluent samples were later assayed for DA using high performance liquid chromatography with electrochemical detection (HPLC-EC).

The HPLC-EC system employed a Whatman ODS-3 reverse-phase C-18 column and a LC. 4 amperometric detector with a glassy carbon electrode (BioAnalytical Systems, Lafayette, IN). The mobile phase was composed of 3 parts of $0.1 \mathrm{M}$ citric acid, 2 parts $0.1 \mathrm{M} \mathrm{NaHPO}_{4}, 0.0004 \%$ sodium octyl sulfate, and 15\% methanol (apparent $\mathrm{pH}$ 3.35). The mobile phase was pumped at $1 \mathrm{ml} / \mathrm{min}$ and continuously recycled. The detector potential was set at $+0.72 \mathrm{~V}$ versus an $\mathrm{Ag} / \mathrm{AgCl}$ reference electrode. Ten microliter samples of the supernatant were assayed with a sensitivity of $10 \mathrm{pg} \mathrm{DA}$ at an amplifier potential of $2 \mathrm{nA} / \mathrm{V}$.

OVX female rats were used in this experiment for the following reasons: (a) in our behavioral experiments we found that OVX females show a longlasting (3-4 weeks) sensitization of rotational behavior following a single injection of $1.25 \mathrm{mg} / \mathrm{kg}$ of AMPH (see Results); (b) the AMPH-stimulated release of DA from striatal tissue obtained from OVX rats is much lower than from intact females or males (6). We thought this lower rate of DA release would make it easier to see a small increase; and (c) we wanted to avoid the large fluctuations in striatal DA release seen across the estrous cycle in intact female rats (6).

\section{RESULTS}

Experiment 1. Change in AMPH-induced rotational behavior between 2 test sessions separated by 3-4 weeks in intact female, OVX female, CAST male and intact male rats

Following an injection of AMPH the majority of animals turned predominantly in one direction, as reported earlier by Glick and his colleagues ${ }^{14}$. Of a total of 37 female rats 19 turned predominantly to the right and 18 predominantly to the left (intact females - 10 right (R), 11 left (L); OVX - 9R, 7L). However, of a total of 50 male rats 32 turned left 
and only 18 right $\left(\chi^{2}=3.92, P<0.05\right.$; intact males - 10R, 17L; CAST - 8R, 15L). Some animals made very few net rotations. The analysis of difference scores which follows was conducted for the 87 'lateralized' rats which: (a) made 5 or more net rotations during each test session: (b) had a percent dominance score of 70 or greater during each test session; and (c) turned in the same dominant direction during both test sessions. The 'non-lateralized' rats are discussed separately (see below).

To determine if there was a change in rotational behavior between the first (1st) and second ( 2 nd) test sessions difference scores were obtained for all animals by subtracting the number of net rotations made during the 1st test session from those made during the 2 nd. If each group made, on the average, the same number of net rotations during each test session this difference score would equal zero. A positive number indicates more net rotations were made during the 2 nd test session than during the 1 st, and a negative number that more net rotations were made during the 1 st test session than during the 2 nd.

Fig. 1 shows the difference in net rotations between two test sessions separated by 3-4 weeks for all 4 groups of animals. A $t$-test was conducted for each group to test the hypothesis that the difference between the two test sessions equals zero (all tests

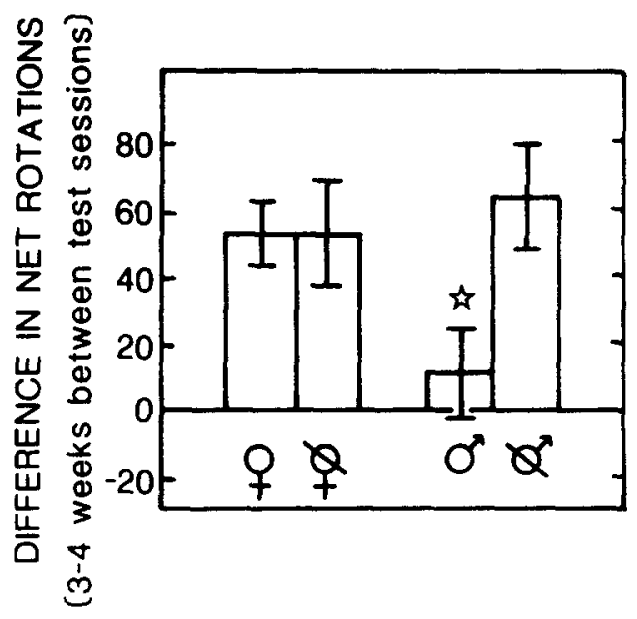

Fig. 1. The mean ( \pm S.E.) difference in the number of net rotations produced by AMPH between two 60-min test sessions separated by 3-4 weeks. From left to right the groups consist of intact females, OVX females, intact males and CAST males. All groups except the intact males made significantly more net rotations during the 2 nd test session than during the 1st (see text). two-tailed). The difference scores for the intact females, OVX females and CAST males are all significantly greater than zero $(t=5.01,3.18$ and 3.78 , and $P<0.001,0.006$ and 0.001 , respectively). These 3 groups made approximately 50-60 more net rotations during the 2 nd test session than during the Ist. This represents an average percent increase of $105 \%$ for these 3 groups. In contrast, the intact males did not show any difference in net rotations between the 1 st and 2 nd test sessions $(t=0.87$; see Fig. 1).

The same analysis as just described was also conducted for total rotations and extra quarter turns (XQT). Exactly the same pattern of results was found for total rotations as for net rotations. However, a very different pattern was found for XQT. The difference scores for XQT did not differ from zero for any of the 4 groups. Thus, all the animals made the same number of XQT during the 2nd test session as during the 1 st.

A simple inspection of the data presented in Fig. I would lead one to conclude that a single injection of AMPH resulted in a long-term enhancement (sensitization) of rotational behavior in intact females, OVX females and CAST males, but not in intact males. However, an analysis of the difference in net rotations between the 1st and 2 nd test sessions for each 5 min interval (see Fig. 2) reveals the results are more complex than is evident from Fig. 1. Fig. 2 shows that the intact females, OVX females and CAST males made more net rotations during the 2nd test session than during the 1st test session for every 5 min interval following AMPH treatment. A one-way analysis of variance for repeated measures across time was conducted on the difference scores for each of the 4 groups. There were no significant changes in the difference scores across time for the intact females, OVX females and CAST males. However, the intact males showed a significant decline in their difference scores across time $(F=$ 5.91, $P<0.001$ ).

Comparisons between the 4 groups were made using a profile analysis 29 of all 12 five-minute intervals. An overall profile analysis comparing the 4 curves presented in Fig. 2 was significant $(P<$ 0.004 ), confirming that the 4 curves are not parallel. Subsequent profile analyses were therefore conducted comparing each group with each other 

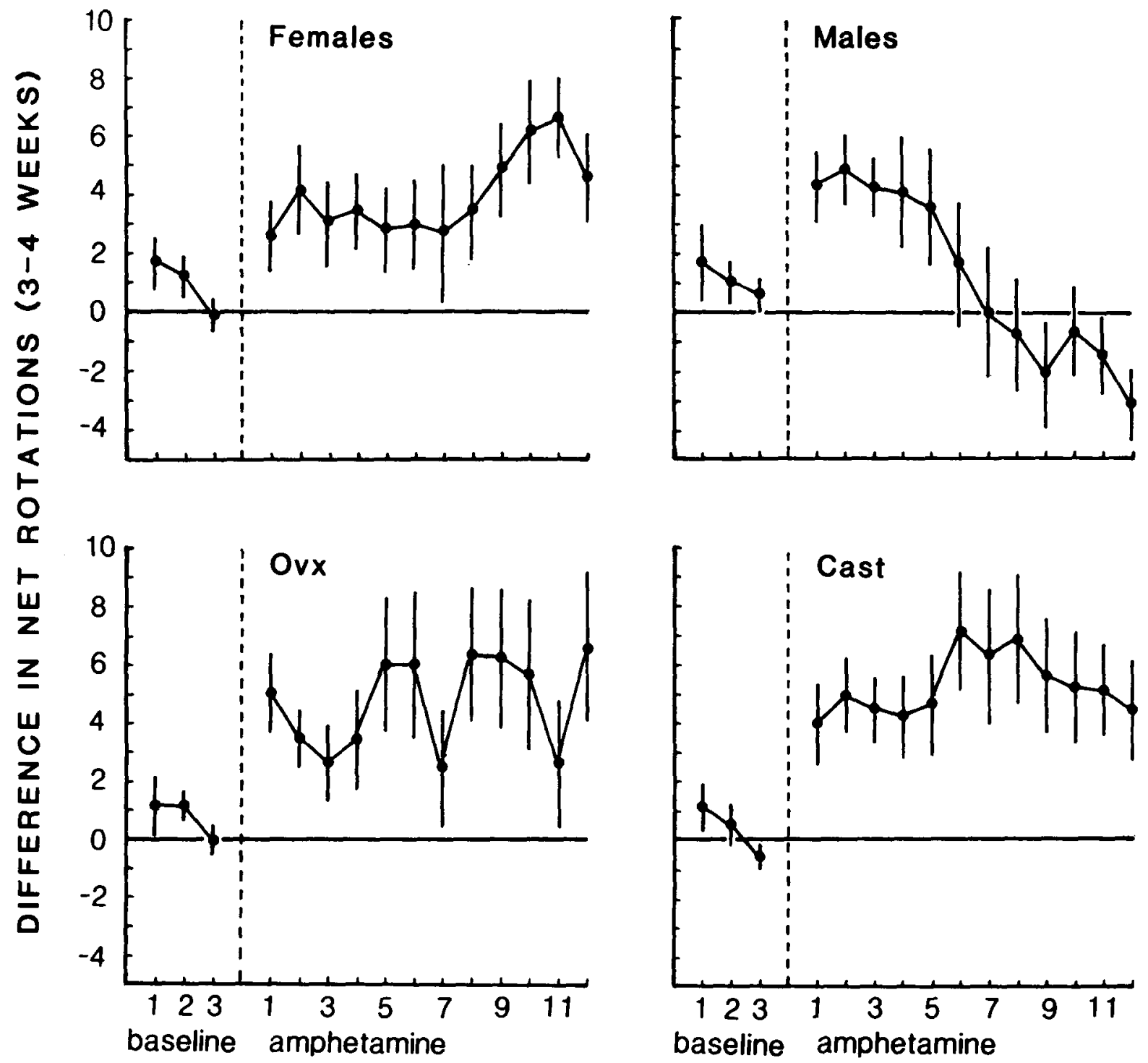

\section{MIN INTERVALS}

Fig. 2. Time course of the changes in the number of net rotations produced by AMPH between two test sessions separated by 3-4 weeks. Each data point represents the mean ( \pm S.E.) difference in net rotations for one 5-min interval. There are 3 habituation intervals represented to the left of the vertical dashed line and 12 intervals after AMPH administration represented to the right of the dashed line (see text for a description of these results).

group. These comparisons showed that the profile for the intact males differs from that of all other groups $(P<0.038-0.001)$. None of the other 3 groups differ from each other, except the profile of the OVX females is not parallel with that of the CAST males $(P<0.02)$.

Since the difference scores for the intact males decreased over time additional profile analyses were conducted comparing the profiles of the 4 groups over the initial 6 intervals ( $30 \mathrm{~min}$ ) of the test session separately from the last 6 intervals of the test session (30 $\mathrm{min}$ ). An overall profile analysis comparing the 4 groups across the initial 6 intervals was not significant. Therefore, these 4 profiles are parallel. Also, the 4 groups do not differ significantly in the level of the difference scores over the first $30 \mathrm{~min}$ of the test sessions (Fig. 2). This suggests the difference between the 4 groups is due entirely to differences which develop during the last $30 \mathrm{~min}$ of the test session. This suggestion is supported by the profile 
analysis on the last 6 intervals. An overall profile analysis of the 4 curves obtained over the last 6 intervals was significant $(P<0.01)$. Further profile analyses between each group showed that the intact males differ from the intact females, OVX females and CAST males $(P<0.005)$. The other groups do not differ from each other, except again, the $O V X$ females have a different profile than the CAST males $(P<0.02)$.

In summary, the impression from Fig. I that intact females, OVX females and CAST males made more net rotations during the 2 nd test session than during the $1 \mathrm{st}$ is confirmed by the more detailed analysis given in Fig. 2. However, it may be misleading to say that the intact males do not show sensitization (Fig. 1). Fig. 2 shows that the intact male did make more net rotations during the 2 nd test session for the first few 5 min intervals after AMPH administration. In contrast to the other groups the intact males did not sustain this enhancement of rotational behavior over the full 1-h session. In fact, by the last few intervals of the test session negative difference scores were obtained, indicating that the intact males actually made more net rotations during the 1st test session.

An enhancement in the number of rotations between the 1st and 2nd test sessions could result from either a decrease in the number of total rotations made in the non-dominant direction, an increase in the turns to the dominant direction, or both. Table I shows that the increase in net rotations seen in intact females, OVX females and CAST males was largely due to an increase in turns to the dominant side. The difference in the number of full rotations made in the dominant direction between the $1 \mathrm{st}$ and 2 nd test sessions was significantly greater than zero for the intact females, OVX females and CAST males $(t=$ $4.62,3.59,3.71 ; P<0.001,0.003$ and 0.002 , respectively). In the OVX females and CAST males there was no change in the number of full rotations made in the non-dominant direction $(t=1.0$ and 0.9 , respectively), while the intact females actually showed a small, but statistically significant decrease $(t=$ 2.17, $P<0.04$ ). Thus, the behavior of the intact females, OVX females and CAST males was far more lateralized after the 2nd AMPH treatment than after the 1st. In contrast, the intact males showed no change in full rotations to either the

\section{TABLE I}

Mean ( $t$ S.E.) difference in the number of full rotations in the dominant or non-dominant direction between the first test session, and a second test session given $3-4$ weeks later.

A positive value indicates that the animals made more full rotations during the second test session than during the first. A negative value indicates the opposite. Rotational behavior was induced with $1.25 \mathrm{mg} / \mathrm{kg}$ of $\mathrm{AMPH}$.

\begin{tabular}{|c|c|c|}
\hline & $\begin{array}{l}\text { Dominant } \\
\text { direction }\end{array}$ & $\begin{array}{l}\text { Non-dominant } \\
\text { direction }\end{array}$ \\
\hline Intact females & $50.1 \pm 10.8^{* *}$ & $-2.8=1.3 *$ \\
\hline OVX females & $58.1+16.2 * *$ & $3.4 .3 .3 \mathrm{NS}$ \\
\hline Intact males & $10.2+14.1 \mathrm{NS}$ & $-8.1 \quad 1.5 \mathrm{NS}$ \\
\hline CAST males & $62.2 \div 16.8 * *$ & $-0.8 \quad 0.9 \mathrm{NS}$ \\
\hline
\end{tabular}

dominant or non-dominant side between the two test sessions (Table I).

For the striking rise in behavioral sensitivity to AMPH to occur it seems that the animals must be behaviorally lateralized. Eleven intact females, 16 OVX females, 14 CAST males and 13 intact males were not included in the above analysis because they did not meet the criteria outlined in the first paragraph of the results section of this paper (i.e. they were not 'lateralized'). These animals did not show any significant change between the 1 st and 2 nd test sessions on any of the measures taken. Fig. 3 shows

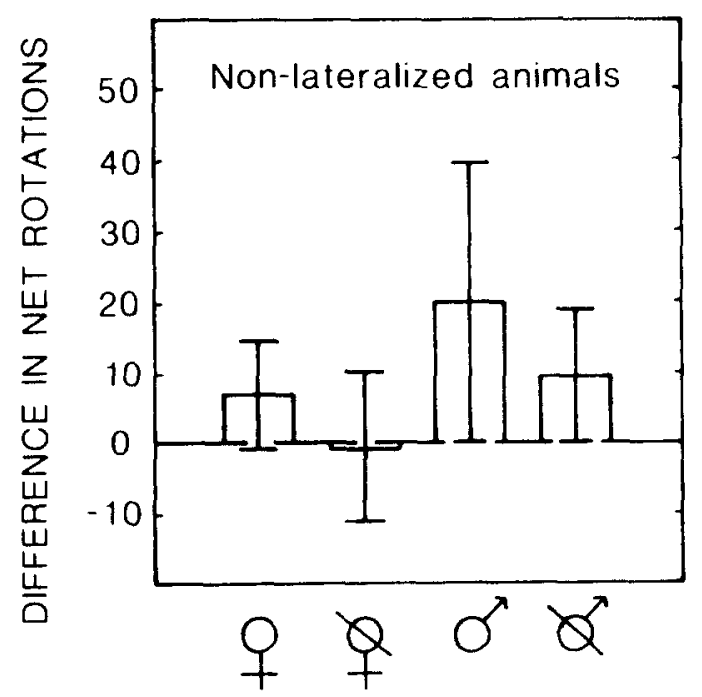

Fig. 3. The difference in the number of net rotations produced by AMPH between two 60 -min test sessions separated by 3-4 weeks in non-lateralized rats (see text). 
the difference in the number of net rotations between the 1st and 2 nd test sessions for these 'nonlateralized' rats. The $t$-tests conducted to determine if these difference scores differ from zero were all non-significant (for intact females, $t=1.0$; OVX females, $t=0.1$; CAST males, $t=1.1$; intact males, $t=1.1$ ). A one-way analysis of variance comparing the 4 groups of non-lateralized animals was also non-significant $(F=0.5)$.

Lastly, a group of 9 female rats were injected with saline during their 1st test session and with AMPH 3 weeks later. When these animals were tested with AMPH 3 weeks after the saline they did not show a significantly greater number of net rotations than did female rats given AMPH during the 1st test session.

\section{Experiment 2. Change in AMPH-induced rotational} behavior between 2 test sessions separated by 7-8 days in intact male and female rats

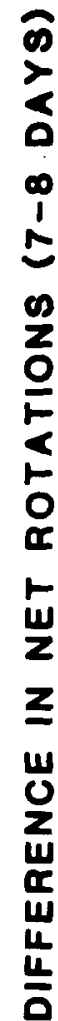

The difference in net rotations between test sessions separated by $7-8$ days for intact male $(n=30)$ and female rats $(\mathrm{n}=28)$ is shown in Fig. 4 . $t$-Tests conducted to determine if the mean difference scores are greater than zero were significant for both males and females (for males, $t=2.77, P<0.01$; females, $t=4.84, P<0.0001$; see Fig. 4A). Thus, both intact males and females made significantly more net rotations during the 2 nd test session than during the 1st. However, the intact females showed a significantly greater increase in net rotations between the two test sessions than did the intact males ( $t$-test on difference between two means, $t==1.97, \mathrm{df}=56 ; P$ $<0.05$; Fig. 4A).

As in Experiment 1, an analysis of the difference scores in net rotations across each 5-min interval revealed an interesting pattern of change across time (Fig. 4B). A profile analysis of the curves plotted in Fig. 4B revealed that the male and female profiles are parallel (i.e. their shapes are not significantly

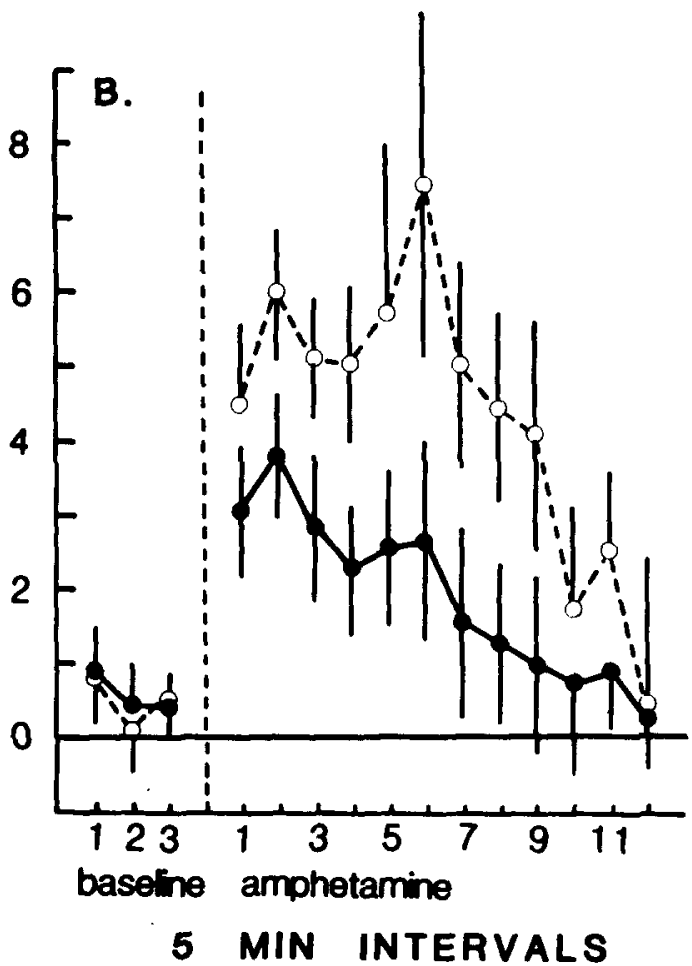

Fig. 4. A: the difference in the mean ( \pm S.E.) number of net rotations produced by AMPH between two 60-min test sessions separated by $7-8$ days in intact female and male rats. Star $=$ males and females differ, $P<0.05$. B: time course of the changes in the number of net rotations produced by AMPH between two test sessions separated by 7-8 days in intact female and male rats. Each data point represents the mean $( \pm$ S.E.) difference for one 5 -min interval. Open circles $=$ intact females; closed circles $=$ intact males (see text). 

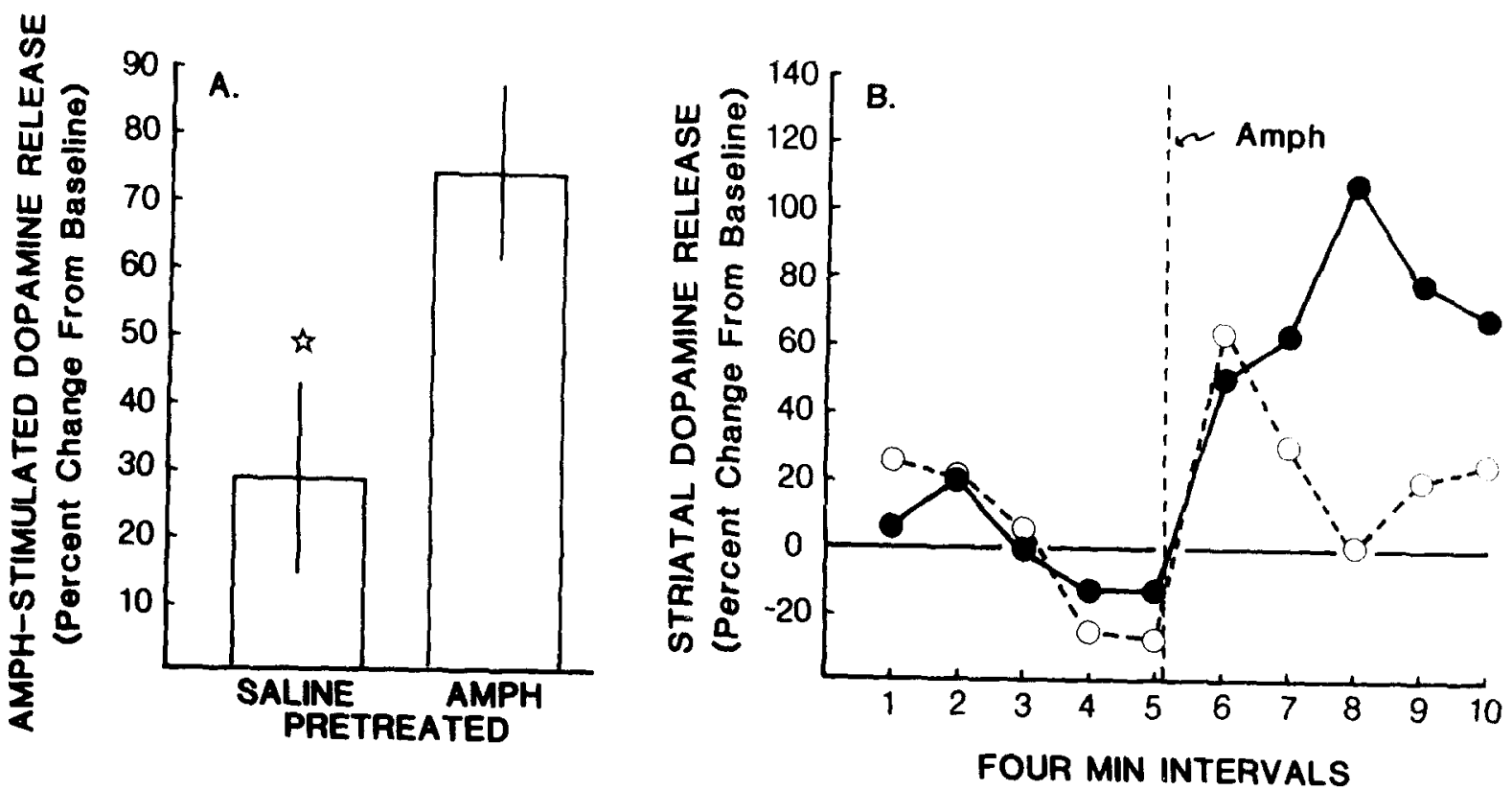

Fig. 5. A: percent change in AMPH-stimulated DA release from striatal tissue in vitro. Saline-pretreated OVX rats were injected with $1.25 \mathrm{ml} / \mathrm{kg}$ of saline and AMPH-pretreated OVX rats with $1.25 \mathrm{mg} / \mathrm{kg}$ of AMPH one month earlier. Star $\ldots$ saline- and AMPH-pretreated groups differ, $P<0.03$. B: time course of the change in DA release from striatal tissue fragments in vitro. AMPH was added to the perifusion medium at the vertical dashed line. Symbols: open circles = saline-pretreated OVX rats; closed circles =- AMPH-pretreated OVX rats. (See text for details.)

different). However, the same analysis resulted in a significant difference in the level of the two curves, the female difference scores being greater than the males' $(P<0.036)$. In addition, one-way analyses of variance for repeated measures across time show that the magnitude of the difference between the two test sessions declines significantly over the 12 fiveminute intervals in both the males and females $(P<$ 0.001 ).

\section{Experiment 3. Changes in AMPH-stimulated dopa-} mine release from striatal tissue in vitro produced $b y$ AMPH pretreatment in vivo

The amount of DA released from striatal tissue was measured for 5 four-minute baseline intervals and for 5 four-minute intervals following a pulse injection of AMPH into the perifusion chamber. All the animals were ovariectomized, but half had been given an i.p. injection of AMPH $(1.25 \mathrm{mg} / \mathrm{kg})$ and half an injection of saline a month prior to being killed ( $\mathrm{n}=8 /$ group). The baseline level of DA released $(\mathrm{pg} / \mathrm{mg} / \mathrm{min})$ into the effluent from striatal tissue was the same in AMPH-pretreated and salinepretreated rats. However, the level of AMPH-stimulated DA release differed in the two groups.
Fig. 5A shows the percent change from baseline in AMPH-stimulated DA release for saline and AMPH-pretreated animals. Baseline is defined as the average level of DA release over the $20 \mathrm{~min}$ baseline period. Both saline- and AMPH-pretreated rats showed an increase in DA release when AMPH was added to the perifusion chamber, but the percent increase was significantly greater in AMPHpretreated rats than in saline-pretreated rats $(t=$ 2.35, $P<0.03$ ). Fig. 5B shows the percent change in $D A$ release across time. It can be seen that during the first interval following the introduction of $A M P H$ into the chamber the striatal tissue from both the saline- and AMPH-pretreated animals showed a comparable increase in DA release. However, the tissue from AMPH-pretreated animals sustained a relatively high level of DA release while the rate of DA release from saline-pretreated tissue declined. A two-way analysis of variance with repeated measures on one factor was performed on the data shown in Fig. 5B. It confirmed that the striatal tissue obtained from AMPH-pretreated rats showed a greater increase in AMPH-stimulated DA release than did striatal tissue obtained from saline-pretreated rats $(F=5.5, P=0.034)$. 


\section{DISCUSSION}

In the experiments reported here we used AMPHinduced rotational behavior as a behavioral index of mesostriatal DA activity to study long-term changes produced by AMPH treatment. We found that a single injection of a relatively small dose of AMPH can greatly enhance the rotational behavior produced by a second injection given 3-4 weeks after the initial treatment in intact female, OVX female and CAST male rats. In contrast, when 3-4 weeks separated the 2 test sessions intact male rats did not show the same pattern of change in AMPH-induced rotational behavior as did the other groups. If only 7-8 days separated the 2 test sessions, intact male and female rats did show a similar pattern of sensitization, but the magnitude of the change was significantly greater in females than males.

There are a number of factors which could account for this long-term facilitation of rotational behavior produced by a single injection of AMPH. It is possible that the initial experience in the test apparatus somehow 'primes' or conditions the animal so that its behavioral response is enhanced upon a second exposure to the apparatus ${ }^{42}$. Segal ${ }^{38}$ has examined experiential factors involved in the progressive augmentation of stereotyped behavior produced by repeated AMPH injections and concludes they cannot account for sensitization (also see refs. 7 and 37). Evidence reported here supports this conclusion. Intact male rats had the same experience as intact female, OVX female and CAST male rats during the first test session, but did not show a similar pattern of sensitization 3-4 weeks later. In addition, a group of female rats injected with saline on the first test session and given AMPH 3 weeks later did not differ from females given AMPH for the first time. This suggests that experience with the apparatus alone is not sufficient to produce sensitization. However, this does not exclude the possibility that conditioning plays some role in the sensitization phenomenon.

Another factor which could account for sensitization is that AMPH or one of its metabolites persists in brain tissue long after the first injection and somehow increases the efficacy of the second injection. Although this possibility has not been ruled out it does not seem likely that it could be responsible for such long term changes as reported here $7,25,38$.

It has been suggested by a number of researchers that the sensitization of stereotyped behavior produced by $\mathrm{CA}$ agonists is in fact due to long-term changes in the functional activity of CA neurons ${ }^{22}$, $27,37,38$. The initial injection of AMPH could alter CA biosynthetic capacity, storage, release, feedback control systems or receptor sensitivity. We report here that a single injection of $1.25 \mathrm{mg} / \mathrm{kg}$ of AMPH in vivo produced a long-lasting (3-5 weeks) enhancement of AMPH-stimulated DA release from striatal tissue in vitro. The dose of AMPH used to produce this enhancement of striatal DA release was the same as that which produced a facilitation of rotational behavior. The enhancement of striatal DA release also lasts at least as long as the enhancement of rotational behavior. Obviously, these changes in the responsiveness of striatal tissue to AMPH could be responsible for the facilitation of rotational behavior. Indeed, long-lasting changes in the release of DA may also be involved in the sensitization of stereotyped behaviors produced by the repeated administration of stimulant drugs. This does not exclude the possibility that changes in other dopaminergic mechanisms are also involved. For example, it has been suggested that sensitization is due to a subsensitivity of DA autoreceptors $1,3,21,27$, 30 . This would be expected to produce an enhancement in DA release. Of course, we cannot rule out the possibility that the actual change(s) takes place in a non-dopaminergic system which modulates DA activity.

The differences between the 4 groups in the pattern of sensitization across the test session are intriguing. The significance of these patterns is not clear, but they may provide clues as to the neurological mechanisms which underlie sensitization. It is possible that the change produced in females by AMPH pretreatment is simply more robust than that in males, and this accounts for the ability of females to sustain their enhanced level of rotational behavior across all twelve 5-min intervals. It is also possible that the differing patterns represent changes in two distinct processes. Changes in one process (e.g. DA release, receptors, etc.) may result in enhanced rotational behavior during the initial few 5-min intervals after AMPH administration, and changes in a sec- 
and distinct process may be responsible for the sustained sensitization seen in females, but not males, during the last few 5-min intervals. Females showed a greater enhancement of rotational behavior during the last few 5-min intervals when 3-4 weeks separated the 2 test sessions than when only 7-8 days separated the 2 test sessions. Therefore, the process responsible for the sensitization observed during the latter part of a test session may take a considerable ( 1 week) amount of time to develop. Further experiments are required to resolve these issues.

The fact that one exposure to AMPH may induce a long-term facilitation of both rotational behavior and AMPH-stimulated DA release raises the question of whether it is necessary to repeatedly administer stimulant drugs over extended periods of time to induce long-lasting changes in neural activity. Sensitization following a single injection of a DA mimetic is not unique to rotational behavior, and has been reported for stereotyped behaviors as well $2,7,12,27$ (also see ref. 26). Receptor hypersensitivity has also been reported following a single injection of a CA antagonist ${ }^{8,9}$. Antelman and Chiodo ${ }^{1}$ recently suggested that the sensitization produced by AMPH or tricyclic antidepressants is actually enhanced if drug injections are widely spaced in time. The idea that drug effects may be greater when drugs are administered infrequently, and that 'drug-free' periods are required for maximal sensitization, has obvious implications for the etiology and pharmacological treatment of psychiatric disorders (e.g. see refs. 1 and 33).

\section{REFERENCES}

1 Antelman, S. M. and Chiodo, L. A., Dopamine autoreceptor subsensitivity: a mechanism common to the treat ment of depression and the induction of amphetamine psychosis, Biol. Psychiatr., 16 (1981) 717-727.

2 Antelman, S. M., Eichler, A. J., Black, C. A. and Kocan, D., Interchangeability of stress and amphetamine in sensitization, Science, 207 (1980) 329-331.

3 Baudry, M., Costentin, J., Marcais, H., Martres, M. P., Protais, P. and Schwartz, J. C., Decreased responsiveness to low doses of apomorphine after dopamine agonists and the possible involvement of hyposensitivity of dopamine 'autoreceptors', Neurosci. Lett., 4 (1977) 203-207.

4 Becker, J. B. and Ramirez, V. D., Dynamics of endogenous catecholamine release from brain fragments of male and female rats, Neuroendocrinology, 31 (1980) 18-25.

5 Becker, J. B., Robinson, T. E. and Lorenz, K. A., Sex
It is intriguing that the pattern of long-term changes differed depending on sex and hormonal condition. Gonadal hormones are known to be intimately involved in controlling the patterns of neural connectivity in the hypothalamus and preoptic area during development ${ }^{15}$. In birds gonadal hormones continue to alter neuronal systems throughout adulthood ${ }^{10,32}$. The perinatal hormonal environment also influences the extent of axonal collateral sprouting in the hippocampus of adult rats $^{28}$. In adult rats gonadal hormones modulate mesostriatal DA activity 6,18 , and there are sex differences in this system $6,35,36$. Therefore, it seems reasonable to speculate that hormones influence the development of long-term changes in the mesostriatal DA system (also see ref. 23). However, the extent of gonadal hormone involvement in this phenomenon remains to be determined. For example, another factor which may contribute to the sex differences reported here is the possibility of sex differences in the lateralization of striatal $\mathrm{DA}^{35}$.

\section{ACKNOWLEDGEMENTS}

We thank L. Conlon and M. Kielb for assistance in testing the animals, B. Therrien for statistical advice concerning the profile analyses, M. Hoy for typing the manuscript, and E. S. Valenstein and J. Woods for their helpful comments on an earlier ve sion. Supported by NINCDS Grant NS16437, Biomedical Research Support Grant RR07050 and NICHHD Fellowship HD05997.

differences and estrous cycle variations in amphetamineelicited rotational behavior, Europ. J. Pharmacol., 80 (1982) 65-72.

6 Becker, J. B. and Ramirez, V. D., Sex differences in the amphetamine stimulated release of catecholamines from rat striatal tissue in vitro, Brain Research, 204 (1981) 361-372.

7 Browne, R. G. and Segal, D. S., Metabolic and experiential factors in the behavioral response to repeated amphetamine, Pharmacol. Biochem. Behav., 6 (1977) 545-552.

8 Christensen, A. V., Acute and delayed effect of a single dose of a neuroleptic drug, Acta physiol. Scand., Suppl. 396 (1973) 78.

9 Christensen. A. V. and Moller-Nielsen, 1., Dopaminergic supersensitivity: influences of dopamine agonists, cholinergics, anticholinergics and drugs used for the treatment of tardive dyskinesia, Psychopharmacologia, 62 
(1979) 111-116.

10 DeVoogd, T. and Nottebohm, F., Gonadal hormones induce dendritic growth in the adult avian brain, Science, 214 (1981) 202-204.

11 Echols, S. D., Circling of mice bearing unilateral striatal lesions: development of increased response to D-amphetamine, Life Sci., 21 (1977) 563-568.

12 Ellison, G. and Morris, W., Opposed stages of continuous amphetamine administration: parallel alterations in motor stereotypies and in vivo spiroperidol accumulation, Europ. J. Pharmacol., 74 (1981) 207-214.

13 Gallardo, E. and Ramirez, V. D., A method for the superfusion of rat hypothalami: secretion of luteinizing hormone-releasing hormone (LH-RH), Proc. Soc. exp. Biol., Med., 155 (1977) 79-84.

14 Glick, S. D., Jerussi, T. P. and Zimmerberg, B., Behavioral and neuropharmacological correlates of nigrostriatal asymmetry in rats. In S. Harnad et al. (Eds.), Lateralization in the Nervous System, Academic Press, New York, 1977, pp. 213-249.

15 Greenough, W. T., Carter, C. S., Steerman, C. and DeVoogd, T. J., Sex differences in dendritic patterns in hamster preoptic area, Brain Research, 126 (1977) 63-72.

16 Greenstein, S. and Glick, S. D., Improved automated apparatus for recording rotation (circling behavior) in rats and mice, Pharmacol. Biochem. Behav., 3 (1975) 507-510.

17 Howlett, D. R. and Nahorski, S. R., Acute and chronic amphetamine treatments modulate striatal dopamine receptor binding sites, Brain Research, 161 (1979) 173-178.

18 Hruska, R. E. and Silbergeld, E. K., Increased dopamine receptor sensitivity after estrogen treatment using the rat rotation model, Science, 208 (1980) 1466-1468.

19 Jackson, D. M., Bailey, R. C., Christie, M. J., Crisp, E. A. and Skerritt, J. H., Long-term D-amphetamine in rats: lack of a change in post-synaptic dopamine receptor sensitivity, Psychopharmacology, 73 (1981) 276-280.

20 Jerussi, T. P. and Glick, S. D., Amphetamine-induced rotation in rats without lesions, Neuropharmacology, 13 (1974) 283-286.

21 Kaneno, S. and Shimazono, Y., Decreased in vivo [ $\left[{ }^{3} \mathrm{H}\right]-$ spiroperidol binding in rat brain after repeated methamphetamine administration, Europ. J. Pharmacol., 72 (1981) 101-105.

22 Klawans, H. L. and Margolin, D. J., Amphetamineinduced dopaminergic hypersensitivity in guinea pigs, Arch. gen. Psychiatr., 32 (1975) 725-732.

23 Koller, W. C., Winer, W. J., Klawans, H. L. and Nausieda, P. A., Influence of female sex hormones on neuroleptic-induced behavioral supersensitivity, Neuropharmacology, 19 (1980) 387-391.

24 Kuczenski, R. and Leith, N. J., Chronic amphetamine: is dopamine a link in or a mediator of the development of tolerance and reverse tolerance? Pharmacol. Biochem. Behav., 15 (1981) 405-413.

25 Kuhn, C. M. and Schanberg, S. M., Distribution and metabolism of amphetamine in tolerant animals. In E. H. Ellinwood, Jr. and M. M. Kilbey (Eds.), Cocaine and Other Stimulants, Plenum, New York, 1977, pp. 161-177.

26 Magos, L., Persistence of the effect of amphetamine on stereotyped activity in rats, Europ. J. Pharmacol., 6 (1969) 200-201.
27 Martres, M. P., Costentin, J., Baudry, M., Marcais, H., Protais, P. and Schwartz, J. C., Long-term changes in the sensitivity of pre- and post-synaptic dopamine receptors in mouse striatum evidenced by behavioral and biochemical studies, Brain Research, 136 (1977) 319-337.

28 Milner, T. A. and Loy, R., Organizational effects of sex steroids on sympathetic axonal sprouting into the hippocampus, Soc. Neurosci. Abstr., 7 (1981) 288.

29 Morrison, F. S., Multivariate Statistical Methods, McGraw-Hill, New York, 1967.

30 Muller, P. and Seeman, P., Presynaptic subsensitivity as a possible basis for sensitization by long-term dopamine mimetics, Europ. J. Pharmacol., 55 (1979) 149-157.

31 Nelson, L. R. and Ellison, G., Enhanced stereotypies after repeated injections but not continuous amphetamines, Neuropharmacology, 17 (1978) 1081-1084.

32 Nottebohm, F., Testosterone triggers growth of brain vocal control nuclei in adult female canaries, Brain Research, 189 (1980) 429-436.

33 Post, R. M., Intermittent versus continuous stimulation: effect of time interval on the development of sensitization or tolerance, Life Sci., 26 (1980) 1275-1282.

34 Robertson, H. A., Effect of chronic D-amphetamine or

$\beta$-phenylethylamine on dopamine binding in rat striatum and limbic system, Soc. Neurosci. Abstr., 5 (1979) 570.

35 Robinson, T. E., Becker, J. B. and Ramirez, V. D., Sex differences in amphetamine-elicited rotational behavior and the lateralization of striatal dopamine in rats, Brain Res. Bull., 5 (1980) 539-545.

36 Robinson, T. E., Camp, D. M., Jacknow, D. S. and Becker, J. B., Sex differences and estrous cycle dependent variation in rotational behavior elicited by electrical stimulation of the mesostriatal dopamine system, Behav. Brain Res., in press.

37 Schwartz, J. C., Costentin, J., Martres, M. P., Protais, P. and Baudry, M., Modulation of receptor mechanisms in the CNS: hyper- and hyposensitivity to catecholamines, Neuropharmacology, 17 (1978) 665-685.

38 Segal, D. S., Behavioral and neurochemical correlates of repeated D-amphetamine administration, Advanc. Biochem. Psychopharmacol., 13 (1975) 247-262.

39 Segal, D. S. and Mandell, A. J., Long-term administration of amphetamine: progressive augmentation of motor activity and stereotypy, Pharmacol. Biochem. Behav., 2 (1974) 249-255.

40 Segal, D. S., Weinberger, S. B., Cahill, J. and McCunney, S. J., Multiple daily amphetamine administration: behavioral and neurochemical alterations, Science, 207 (1980) 904-907.

41 Short, P. H. and Shuster, L., Changes in brain norepinephrine associated with sensitization to D-amphetamine, Psychopharmacology, 48 (1976) 59-67.

42 Tilson, H. A. and Rech, R. H., Conditioned drug effects and absence of tolerance to $D$-amphetamine induced motor activity, Pharmacol. Biochem. Behav., 1 (1973) 149-153.

43 Ungerstedt, U., Striatal dopamine release after amphetamine or nerve degeneration revealed by rotational behavior, Acta physiol. scand., 82, Suppl. 367 (1971) 49-68.

44 Wallach, M. B. and Gerson, S., Sensitization to amphetamines, Psychopharmacol. Bull., 7 (1971) 30-31. 